\title{
Is analog preoperative planning still applicable? -comparison of accuracy of analog and computer preoperative planning methods in total hip arthroplasty
}

\author{
Julian Dutka, Slawomir Kiepura, Mateusz Bukowczan \\ Department of Orthopedic and Trauma Surgery in Zeromski's Memorial Specialistic Hospital, Krakow, Poland \\ Contributions: (I) Conception and design: All authors; (II) Administrative support: J Dutka; (III) Provision of study materials or patients: All authors; (IV) \\ Collection and assembly of data: S Kiepura, M Bukowczan; (V) Data analysis and interpretation: All authors; (VI) Manuscript writing: All authors; (VII) \\ Final approval of manuscript: All authors. \\ Correspondence to: Slawomir Kiepura, MD. Department of Orthopedic and Trauma Surgery in Zeromski’s Memorial Specialistic Hospital, os. Na \\ Skarpie 66, 31-913 Krakow, Poland. Email: slawkie@wp.pl.
}

\begin{abstract}
Background: Preoperative planning is an integral part of total hip arthroplasty and has a significant impact on surgical technique and clinical outcome. The variety of types and sizes of endoprosthesis components makes the procedure more demanding and generates a need for accurate preoperative planning. The objective of this study was to analyze an analog method of preoperative planning of primary total hip arthroplasty based on templates overlaying on preoperative radiograms and compare its accuracy for predicting the size, both the stem and cup, with computer planning methods.

Methods: A retrospective cohort study based on 360 X-ray images of hip joints in 348 patients qualified for total hip arthroplasty between 2018 and 2019. The study group consisted of 136 men and 212 women, with an average age of 65 years (56 to 85 years). Material included both cementless and cemented endoprostheses. Results: In the analyzed material, the accuracy of cup planning using the analog method was $85 \%(\mathrm{P}<0.001)$ and $77 \%(\mathrm{P}<0.001)$ in the planning of stem size. However, using the computer method, planning accuracy was $82 \%(\mathrm{P}<0.001)$ for the cup and $72 \%(\mathrm{P}<0.001)$ for the stem.

Conclusions: Both methods of preoperative planning remain effective. The analog method of preoperative planning is simple, precise, and repeatable in choosing the type and size of endoprosthesis components with an accuracy of $85 \%$ and $77 \%$ for the cup and stem respectively. The accuracy of planning depends on the type of endoprosthesis and in the case of the cemented endoprosthesis, it is lower than in cementless.
\end{abstract}

Keywords: Preoperative planning; templating; total hip arthroplasty (THA); cup; stem

Submitted Nov 16, 2020. Accepted for publication Feb 28, 2021.

doi: 10.21037/atm-20-7489

View this article at: http://dx.doi.org/10.21037/atm-20-7489

\section{Introduction}

Preoperative planning is an integral part of total hip arthroplasty (THA) and has a significant impact on surgical technique and clinical outcome. Accurate planning enables the proper selection of component types and sizes in terms of the etiology of degenerative changes, pathological anatomy of the joint, and quality of bone stock. The variety of types and sizes of endoprosthesis components has increased, which makes the procedure more demanding and generates a need for accurate preoperative planning. Choosing the correct implant type and size is crucial for a good final outcome. Inappropriate preoperative planning is the main reason for technical errors in endoprosthesis implantation. Correct acetabular implantation requires reconstruction of the central point of joint rotation, as well as correct inclination and depth of the acetabular component. Correct stem implantation should take into account height, axial rotation, and offset. 
Table 1 Demographic data and the number of examined groups qualified for individual implantation types of endoprostheses

\begin{tabular}{lccccc}
\hline Sex/endoprosthesis type & Alloclassic/Allofit & C-Stem/Ogee & ESOP/HIP\&Go & Exeter/Exeter & Total \\
\hline Average age (in years) & 62 & 78 & 64 & 82 & 65 \\
Female & 63 & 43 & 55 & 42 & 31 \\
Male & 50 & 25 & 97 & 82 & 148 \\
Total & 113 & 68 & 360 \\
\hline
\end{tabular}

Preoperative planning is based on digital X-ray (DXR) images of hip joints scaled through the use of a standard size marker. Analog planning uses templates while computer planning uses dedicated computer software. The effectiveness of the above-mentioned methods may vary among parameters such as accuracy of chosen type and size of the endoprosthesis, planning effort, and total cost.

The objective of this study is to compare the accuracy of preoperative planning in THA using the analog method based on templates with a computer method based on digital software-MediCAD ${ }^{\circledR}$ Classic 5.5.0.8. Therefore, we conducted a comparative, retrospective cohort study based on the prespecified hypothesis according to which broadly used analog preoperative planning is still applicable in clinical practice. We present the following article in accordance with the STROBE reporting checklist (available at http://dx.doi.org/10.21037/atm-20-7489).

\section{Methods}

All performed THA were preceded by preoperative planning both analog and digital. This cohort study was based on 360 DXR images of hip joints in 348 consecutive patients qualified for THA between January 2018 and December 2019. All arthroplasty procedures were performed at Zeromski's Memorial Hospital and all participants who met the inclusion criteria stated below were selected from consecutive patients of the Orthopedic Surgery Department. The study group consisted of $136(39.1 \%)$ men and $212(60.9 \%)$ women, with an average age of 65 years.

The inclusion criterion was the presence of unilateral or bilateral idiopathic hip osteoarthritis. The exclusion criteria were any different etiologies of degenerative changes, acetabular dysplasia, femoral neck fracture, history of hip osteotomies, and other joint dysmetria.

The arthroplasty procedures included both cementless [ESOP/Hip\&Go (FH), Alloclassic/Allofit (Zimmer Biomed)] and cemented endoprostheses [Exeter/Exeter
(Stryker), C-Stem/Ogee (DePuy/J\&J)]. All date were collected from medical record, surgical reports, and postoperative X-ray.

Demographic data and number of examined groups qualified for individual implantation types of endoprostheses are shown in Table 1.

Preoperative DXR of hip joints consists of an AP view including $1 / 3$ of the proximal femur with the $X$-ray beam centered on the pubic symphysis and lower limbs in 10 degrees of internal rotation. Standardization of DXR relies on preserving a constant film-focus distance of $100 \mathrm{~cm}$ between the radiation source and detector. All DXRs were captured by the same X-ray unit - Philips Juno DRF. The scaling of DXR was based on a $30-\mathrm{mm}$ radiopaque metal ball (scaling sphere). The marker was attached to the skin in the area of the greater trochanter in order to achieve a similar distance between the radiation source (bulb of the apparatus) and proximal femur, and marker metal ball in relation to the film.

Preoperative planning was performed by two independent researchers for each patient. Surgeries in both groups were exclusively performed by the senior consultant surgeon who operated on the patient on the following day. The researchers also recorded the average time (in minutes) in which they completed the planning procedure.

In the analog method, DXR magnification was adjusted in the Exhibeon 2.7.28 program to achieve the real size of the marker placed on it. Templates in a 1.15:1 scale, each for a dedicated type of endoprosthesis, were used. The markers were measured using a measuring tape located on the templates with an accuracy of $1 \mathrm{~mm}$. Templates were applied to be scaled in such a manner as to choose the appropriate type, size, and localization of planned implantation of the endoprosthesis. The authors named the method as analog according to the generally accepted nomenclature due to the use of analog templates that do not require any digital tools, but only the adjustment of the X-ray magnification. Analog radiograms have been virtually completely replaced by DXRs. Nevertheless, the 


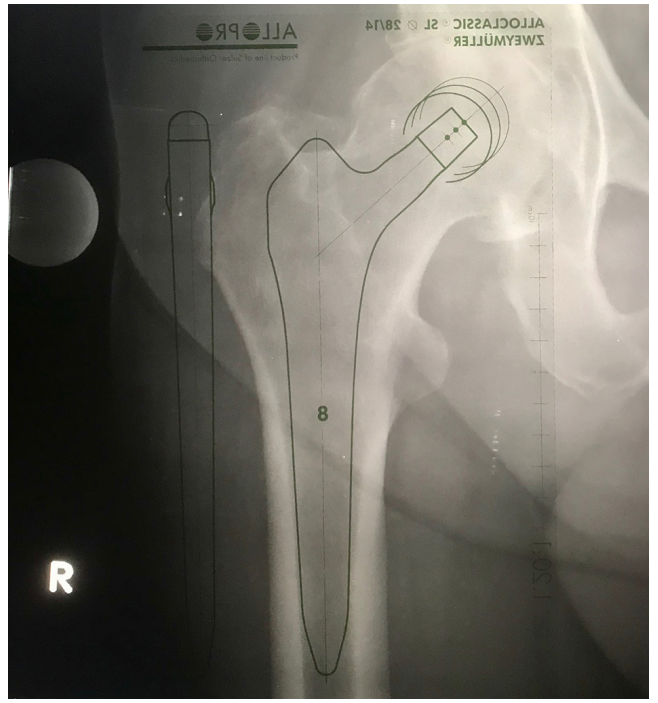

Figure 1 Templating of Alloclassic endoprosthesis. Right hip AP view. AP, anterior-posterior.

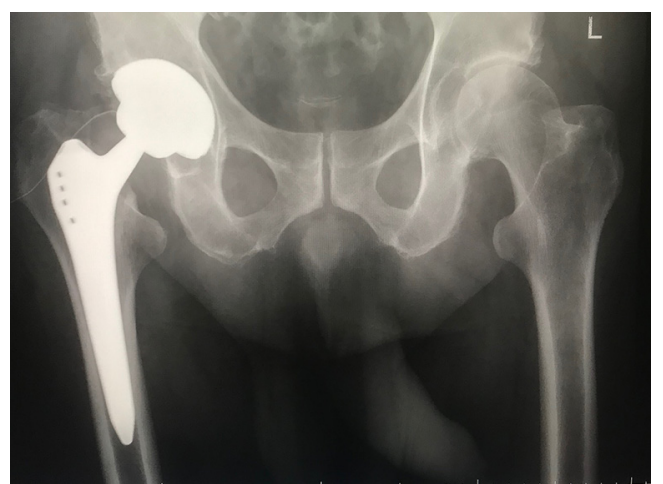

Figure 2 Post-operative X-ray Alloclassic-Allofit. Right hip AP view. $\mathrm{AP}$, anterior-posterior.

technique of this planning remains unchanged. the authors left the name of the analog technique because of the way it is achieved, not the modality used to obtain the $\mathrm{X}$-rays.

The type and size of the cup and stem were determined by applying templates on the DXR to match the outline border of the bony cup and proximal femur segment in order to reconstruct joint anatomy.

Planning appropriate localization of the cup requires the restoration of the joint rotation center, as well as its inclination and depth of placement. The correct size of the acetabular component was determined so that the outline border of the implant on an analog or digital template did not extend beyond the iliosciatic line and Köhler's teardrop.

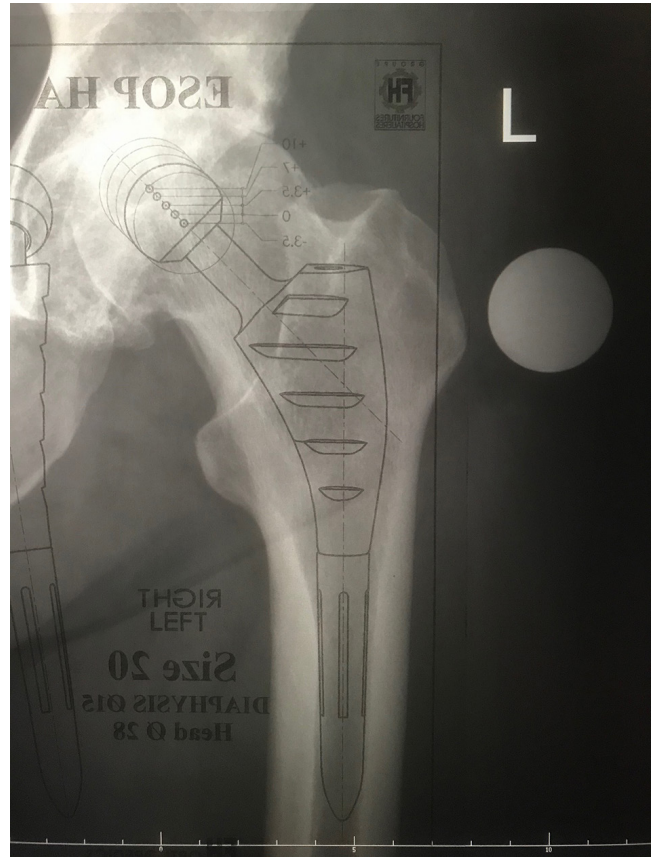

Figure 3 Templating of ESOP endoprosthesis. Left hip AP view. $\mathrm{AP}$, anterior-posterior.

The correct position of the cup and stem was assessed during the operation by measuring the length of the lower extremities. According to the technique, the assessment of the correct depth and orientation of the implant is performed intraoperatively based on anatomical points namely the alignment of the tip of the great trochanter in relation to the center of the implant head.

Planning of stem placement takes into consideration such parameters as the level of femoral neck resection, axial rotation, the height of implantation, and offset as compared to the contralateral side. Selected stem size corresponded to the size on analog or digital templates which were applied to the contour of the inner cortex of the proximal femoral segment on DXR, with an offset similar to that of the contralateral side. Offset is defined as the distance between the axis line of the femoral shaft and the center of rotation (femoral head center). The templating in analog preoperative planning of Alloclassic implantation and postoperative X-ray of the right hip is shown in Figures 1,2. The templating in analog preoperative planning of ESOP implantation and post-operative $\mathrm{X}$-ray of the left hip on Figures 3,4. The templating in analog preoperative planning of C-Stem implantation and post-operative X-ray of the right hip in Figures 5,6. 


\section{Page 4 of 9}

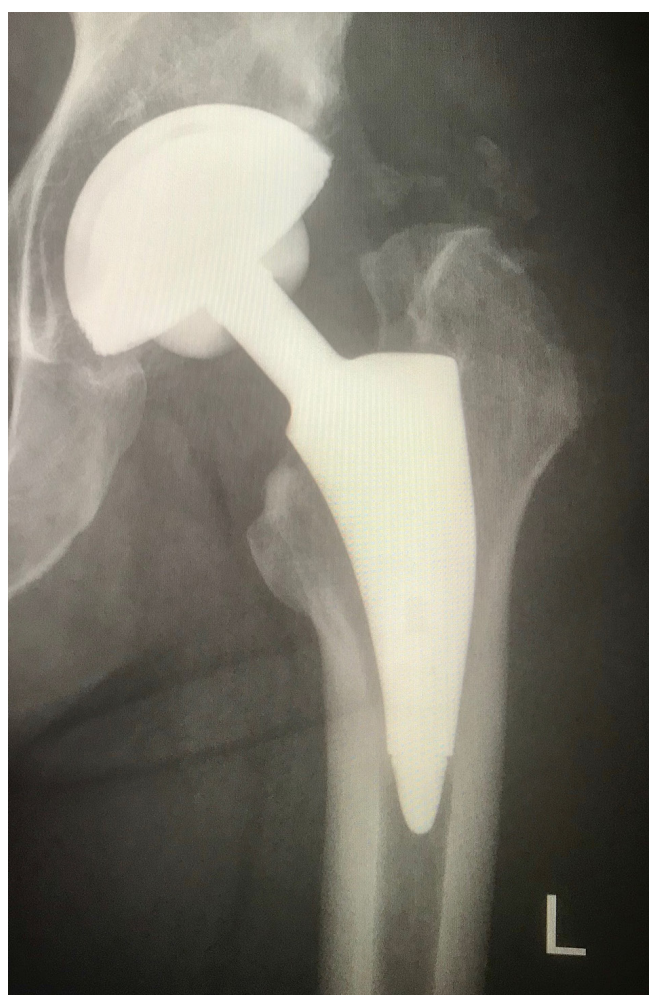

Figure 4 Post-operative X-ray ESOP/Hip\&GO. Left hip AP view. $\mathrm{AP}$, anterior-posterior.

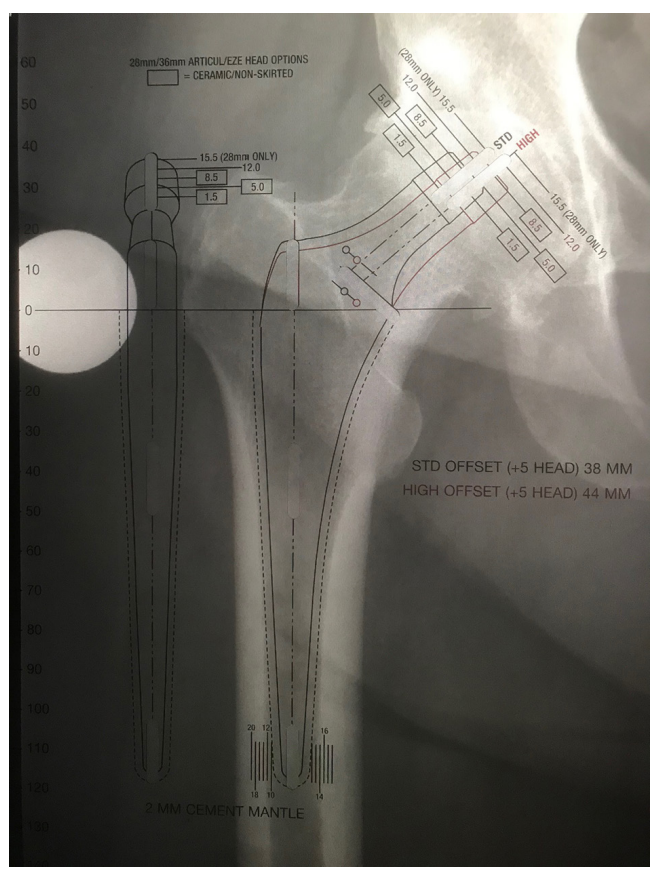

Figure 5 Templating of C-Stem endoprosthesis. Right hip AP view. AP, anterior-posterior.

\section{Dutka et al. Analog preoperative planning is still applicable}

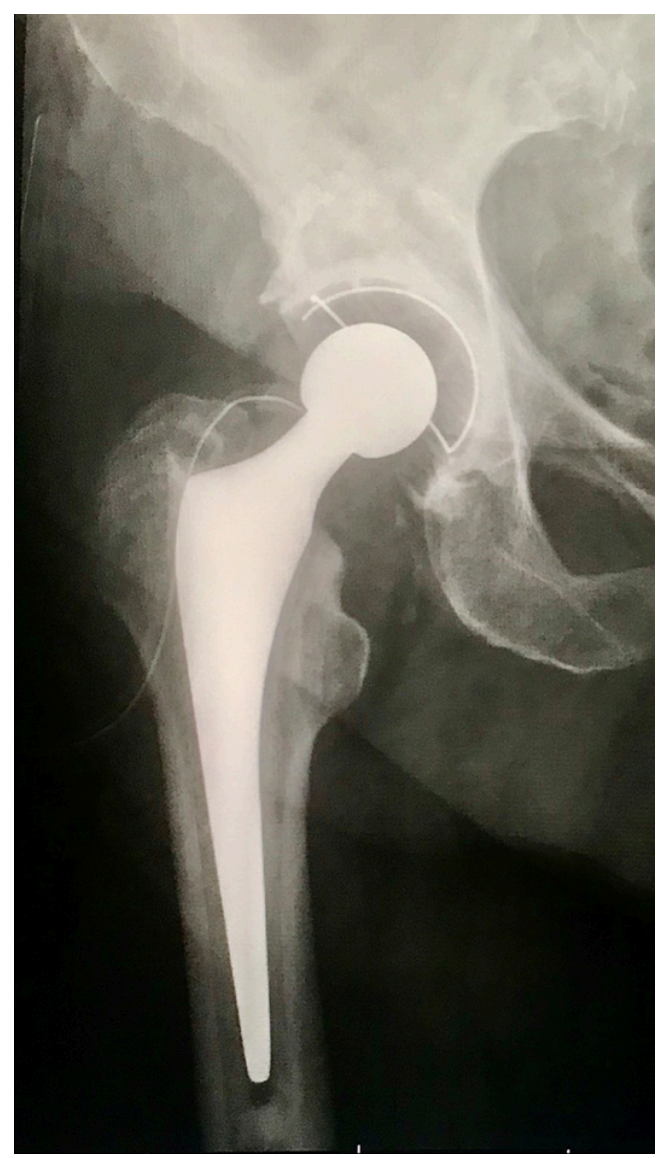

Figure 6 Post-operative X-ray C-Stem-Ogee. Right hip AP view. AP, anterior-posterior.

The digital method was based on performing preoperative planning in MediCAD ${ }^{\circledR}$ Classic 5.5.0.8. This program allows scaling of the image using a marker of known size and applying it to the digital template on DXR in order to determine the type, size, and localization of planned endoprosthesis elements. The choice of implants using the computer method was also based on an analysis of hip jointspecific anatomy and bone stock conditions. The digital planning has been performed using a 17 -inch LCD screen with a resolution of $1.024 \times 768$ pixels. The use of digital preoperative planning follows essentially the same steps as analog one namely preparation of the digital radiograms of the hip, determination the magnification by measuring the metal marker followed by the interposition of the templates dedicated to each type of endoprosthesis then make size and type choice. All the above stages of analysis and planning are carried out using the software tools available.

The objective of this study was to compare the size of 

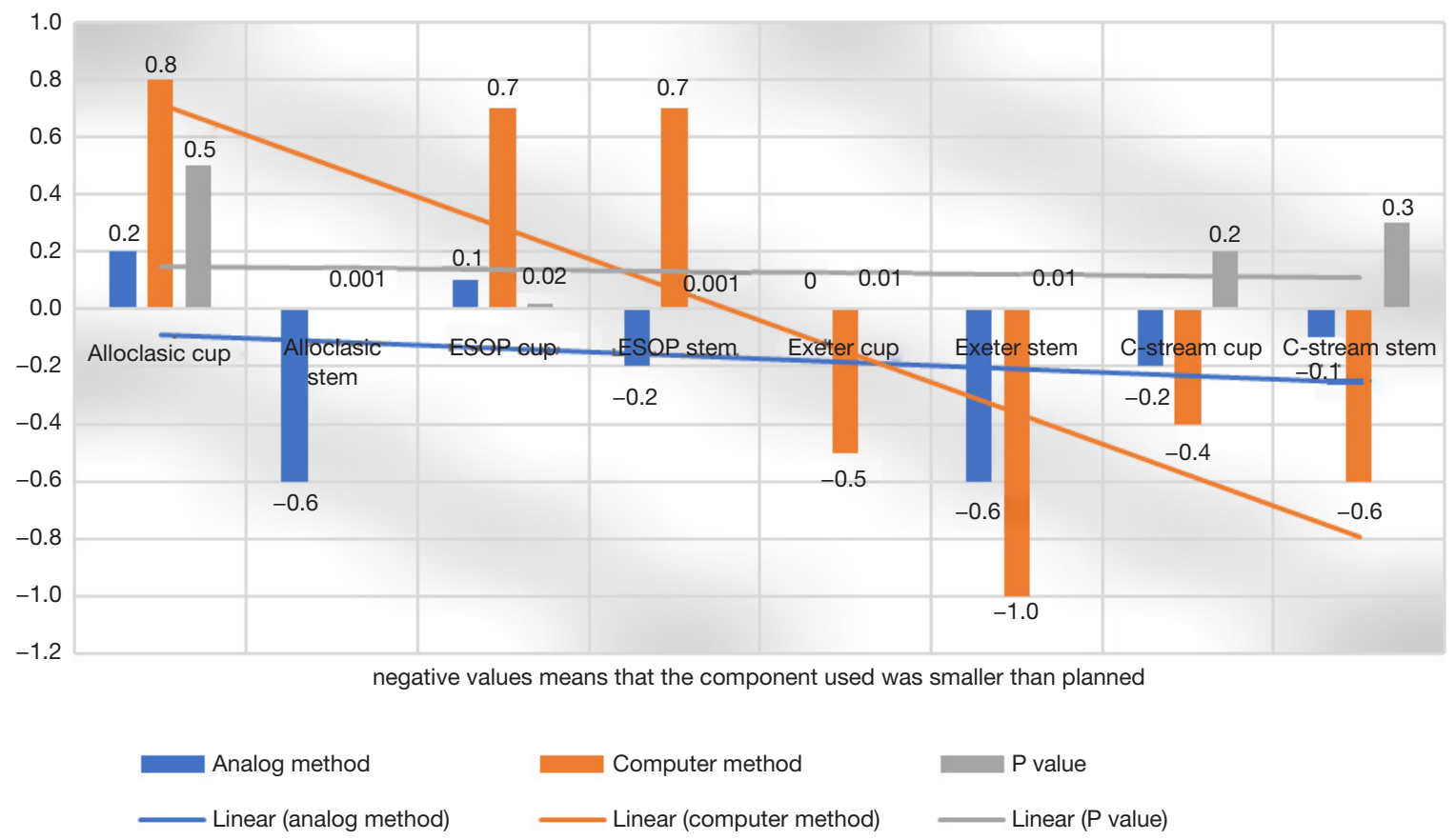

Figure 7 The average value of the difference in the size between planned and actually used cup and stem.

implants planned using both aforementioned methods with the size actually used during the THA procedure. All descriptive variables (size, type of endoprosthesis) for each arthroplasty procedure were collected. There was no missing data. The results of analog and computer planning methods were compared. The same type, size, and localization of planned, and finally implanted endoprosthesis elements were assumed to be a positive result in both preoperative planning methods.

\section{Statistical analysis}

SPSS version 17.0 was used for statistical analysis. Descriptive analysis was performed by determination of values, averages, and standard deviations. Differences were compared using the chi-square test for normal variables. Continuous variables were compared using the $t$-test. A P value of $<0.05$ was set as the significance threshold.

\section{Ethical statement}

The study was conducted in accordance with the Declaration of Helsinki (as revised in 2013). The study was approved by institutional ethics board of Zeromski's Memorial Hospital (No.134/11/2019) and individual consent for this retrospective analysis was waived.

\section{Results}

Among 348 patients enclosed in this study, there were performed 360 arthroplasty procedures -210 cementless and 150 cemented. Twelve patients received bilateral THA. The descriptive data-age, sex, body mass index (BMI) index, an indication of surgery, and time of preoperative planning was not significantly different within the two groups $(\mathrm{P}>0.2)$. In the analyzed material, the accuracy of cup planning using the analog method was $85 \%(\mathrm{P}<0.01)$ and $77 \%(\mathrm{P}<0.01)$ in the planning of stem size. However, using the computer method, planning accuracy was $82 \%$ $(\mathrm{P}<0.01)$ for the cup and $72 \%(\mathrm{P}<0.01)$ for the stem (Table 1).

Analysis of average standard deviation in both methods for the size of the cup and stem resulting from planning and actually used during surgery which corresponding to the average value of the difference in the size between planned and actually used cup and stem is presented in Figure 7.

Statistically significant values were obtained in relation to the planned size of the cup and stem for ESOP/Hip\&Go, Exeter/Exeter, and Alloclassic/Allofit. The highest planning accuracy was found for Allofit/Alloclassic endoprosthesis, $92 \%$ for stem and $90 \%$ for the cup. The lowest planning 
Table 2 Number and the percentage value of used endoprosthesis' components accurate with analog and computer planning

\begin{tabular}{lccc}
\hline Type of endoprosthesis & Component type & Analog method (N/\%) & Computer method (N/\%) \\
\hline Allofit & Cup & $102 / 90.3$ & $100 / 88$ \\
Alloclassic & Stem & $104 / 92$ & $105 / 92$ \\
HIP\&Go & Cup & $75 / 77$ & $79 / 81$ \\
ESOP & Stem & $70 / 72$ & $71 / 73$ \\
Exeter & Cup & $74 / 90$ & $64 / 78$ \\
Exeter & Stem & $70 / 72$ & $70 / 85$ \\
Ogee & Cup & $74 / 90$ & $59 / 87$ \\
C-stem & Stem & $68 / 83$ & $44 / 65$ \\
\hline
\end{tabular}

Table 3 Comparison of summarized accuracy of analog and computer planning methods

\begin{tabular}{lcccc}
\hline Type of endoprosthesis & No. & Stem (N/\%) & Cup (N/\%) & Total mean accuracy (\%) \\
\hline Alloclassic/Allofit & 113 & $106 / 94$ & $104 / 92$ & 93 \\
ESOP/HIP\&Go & 97 & $73 / 75$ & $87 / 90$ & 81 \\
Exeter/Exeter & 82 & $73 / 90$ & $71 / 87$ & 89 \\
C-Stem/Ogee & 68 & $59 / 87$ & $62 / 91$ & 89 \\
Total & 360 & $311 / 86$ & $324 / 90$ & 88 \\
\hline
\end{tabular}

Table 4 Impact of BMI values on accuracy of preoperative planning

\begin{tabular}{lccc}
\hline BMI value & Patient No. & Accuracy of analog method (N/\%) & Accuracy of computer method (N/\%) \\
\hline <18.5: underweight & 67 & $65 / 97$ & $66 / 98$ \\
18.5-24.99: correct value & 183 & $168 / 91$ & $162 / 88$ \\
>25: overweight & 110 & $82 / 74$ & $86 / 78$ \\
\hline
\end{tabular}

accuracy was found for C-Stem/Ogee endoprosthesis. Detailed data of number and the percentage value of used endoprosthesis' components accurate with analog and computer planning are presented in Table 2.

Comparison of summarized accuracy of analog and computer planning methods is presented in Table 3.

Analysis of impact caused by BMI index on preoperative planning showed a decrease in accuracy of implant choice due to increased BMI index in both methods. Data are presented in Table 4.

The average time needed to perform an analog or digital planning procedure for one hip joint was also assessed, and was $7 \pm 2$ minutes in the analog method and $10 \pm 3$ minutes in the computer method.

\section{Discussion}

Preoperative planning is an integral part of the THA procedure and has a significant impact on surgical technique and outcome of treatment. It enables assessment of joint pathology and bone quality, and thus allows the proper choice of type and size of the endoprosthesis. Preoperative planning is based on the analysis of DXR images of hip joints using a metal marker that allows the image to be scaled to the actual size. Currently, both the analog planning method using templates and the computer method using 
dedicated software is in use. The effectiveness of the above methods may differ in such parameters as the accuracy of choice, labor intensity, and related planning costs. Currently, there are numerous types of endoprostheses that differ in the type of biomaterial used, cup or stem geometry, and method of implantation.

In the analyzed data, the accuracy of the analog planning method was $85 \%$ for cups and $77 \%$ for stems. Similar results of the analog planning method were presented by Gonzalez et al. who found an $83 \%$ accuracy for cups and $78 \%$ for stems and for the computer planning method respectively $84.5 \%$ for the cups and $80 \%$ for stems (1). Similar data were obtained in another paper by Holzer et al. with $87 \%$ for cup components and $78 \%$ for stems (2). Osmani et al. after analyzing data obtained when planning THA in 45 patients, did not find a statistically significant difference between analog and digital planning methods (3).

An important factor that may affect results obtained in the planning process is the magnification of DXR size which depends on the distance between the radiation source and detector. One of the main reasons for failure in preoperative planning is the incorrect magnification of DXR without taking into account the constant distance of $100 \mathrm{~cm}$ from the X-ray source to the detector (4-7). Another factor that affects planning accuracy is the localization of marker placement. The authors of this study determined the area of the greater trochanter to be the most favorable localization for marker placement because it is easily palpable in most patients. This method of placing the marker allows achieving a similar distance between the femoral head center and marker in relation to the detector, which reduces the risk of potential error in image scaling. The and White undertake similar methodology in their research $(8,9)$.

Stigler $\mathrm{et}$ al. placed the marker in the area of the pubic symphysis-the location of the central beam, which may affect the precision of image scaling because it develops a greater distance between marker and detector compared to the center of the femoral head (10).

This study showed a relevant impact of BMI index values in planning accuracy. In the high BMI index (>25) patient group, the accuracy of both planning methods significantly decreased. This is due to the difficulty of precisely placing the marker at the level of the greater trochanter with the consequent change in distance between marker and detector, which results in DXR enlargement. Similar observations were made by Holzer et al. who noticed a decrease in accuracy of planning stem size in overweight patients (2). Kniesel et al. did not find a relationship between the value of BMI index and accuracy of component type and size selection (11).

The accuracy of both planning methods varies significantly depending on the type of endoprosthesis. There was a difference in accuracy for both the cup and stem components. In general, significantly higher accuracy was noted for cementless implants, especially for Alloclassic stem and Allofit cup, with the lowest accuracy for Hip\&Go cup and ESOP stem. The et al., after analyzing 173 cases, found $66 \%$ accuracy for cementless stems, and $52 \%$ for cup components (12). In the case of cemented components, lower accuracy of preoperative planning was obtained in both methods. The study showed planning accuracy of $78 \%$ for cups and $86 \%$ for stems. Bertz et al. after analyzing 129 patients with cemented or hybrid endoprostheses, noted $95 \%$ accuracy of stem planning, and $94 \%$ in the cup (13). These results were not confirmed in the authors' study. According to our study, the above difference in planning accuracy is due to the difficulty in obtaining the thickness of the planned cement mantle, in which the cup and stem are implanted.

The authors emphasized that the analog method is a challenge for the planner because it is burdened with the risk of more errors in the technique although the results may be similar. By comparing both methods of preoperative planning, the analog method remains effective and competitive to the computer method. This is confirmed by results showing slightly higher accuracy of the analog method. Undoubtedly, the computer method is definitely progressive and logistically sound in the age of widespread computerization and digitization. However, it is associated with the high cost of purchasing and updating software, and thus limits the number of available computer workstations for planning. On the other hand, in the authors' opinion, the analog method requires more experience and accuracy of each planning step. Similar observations were made by Petretta et al. who demonstrated an advantage of the analog planning method over digital (14).

\section{Conclusions}

(I) The analog method of preoperative planning is precise, repeatable, and easily accessible in choosing the type and size of endoprosthesis components.

(II) The accuracy of planning depends on the type of endoprosthesis-in the case of cemented one is lower than in cementless.

(III) With increased BMI, planning precision in both 
methods decreases.

(IV) The workload and planning time does not differ significantly in both methods.

(V) The computerized planning method requires a greater cost of purchasing licenses and updating software.

\section{Acknowledgments}

Funding: None.

\section{Footnote}

Reporting Checklist: The authors have completed the STROBE reporting checklist. Available at http://dx.doi. org/10.21037/atm-20-7489

Data Sharing Statement: Available at http://dx.doi. org/10.21037/atm-20-7489

Conflicts of Interest: The authors have completed the ICMJE uniform disclosure form (available at http://dx.doi. org/10.21037/atm-20-7489). The authors have no conflicts of interests to declare.

Ethical Statement: The authors are accountable for all aspects of the work in ensuring that questions related to the accuracy or integrity of any part of the work are appropriately investigated and resolved. The study was conducted in accordance with the Declaration of Helsinki (as revised in 2013). The study was approved by institutional ethics board of Zeromski's Memorial Hospital (No. 134/11/2019) and individual consent for this retrospective analysis was waived.

Open Access Statement: This is an Open Access article distributed in accordance with the Creative Commons Attribution-NonCommercial-NoDerivs 4.0 International License (CC BY-NC-ND 4.0), which permits the noncommercial replication and distribution of the article with the strict proviso that no changes or edits are made and the original work is properly cited (including links to both the formal publication through the relevant DOI and the license). See: https://creativecommons.org/licenses/by-nc-nd/4.0/.

\section{References}

1. González Della Valle A, Slullitel G, Piccaluga F, et al.
The precision and usefulness of preoperative planning for cemented and hybrid primary total hip arthroplasty. J Arthroplasty 2005;20:51-8.

2. Holzer LA, Scholler G, Wagner S, et al. The accuracy of digital templating in uncemented total hip arthroplasty. Arch Orthop Trauma Surg 2019;139:263-8.

3. Osmani FA, Thakkar S, Ramme A, et al. Variance in predicted cup size by 2 -dimensional vs 3-dimensional computerized tomography-based templating in primary total hip arthroplasty. Arthroplast Today 2017;3:289-93.

4. Bayne CO, Krosin M, Barber TC. Evaluation of the accuracy and use of $x$-ray markers in digital templating for total hip arthroplasty. J Arthroplasty 2009;24:407-13.

5. White SP, Bainbridge J, Smith EJ. Assessment of magnification of digital pelvic radiographs in total hip arthroplasty using templating software. Ann R Coll Surg Engl 2008;90:592-6.

6. Kulkarni A, Partington P, Kelly D, et al. Disc calibration for digital templating in hip replacement. J Bone Joint Surg Br 2008;90:1623-6.

7. Miashiro EH, Fujiki EN, Yamaguchi EN, et al. Preoperative planning of primary total hip arthroplasty using conventional radiographs. Rev Bras Ortop 2014;49:140-8.

8. The B, Diercks RL, van Ooijen PM, et al. Comparison of analog and digital preoperative planning in total hip and knee arthroplasties. A prospective study of 173 hips and 65 total knees. Acta Orthop 2005;76:78-84.

9. White SP, Shardlow DL. Effect of introduction of digital radiographic techniques on pre-operative templating in orthopaedic practice. Ann R Coll Surg Engl 2005;87:53-4.

10. Stigler SK, Müller FJ, Pfaud S, et al. Digital templating in total hip arthroplasty: Additional anteroposterior hip view increases the accuracy. World J Orthop 2017;8:30-5.

11. Kniesel B, Konstantinidis L, Hirschmüller A, et al. Digital templating in total knee and hip replacement: an analysis of planning accuracy. Int Orthop 2014;38:733-9.

12. The B, Verdonschot N, van Horn JR, et al. Digital versus analogue preoperative planning of total hip arthroplasties: a randomized clinical trial of 210 total hip arthroplasties. J Arthroplasty 2007;22:866-70.

13. Bertz A, Indrekvam K, Ahmed M, et al. Validity and reliability of preoperative templating in total hip 
arthroplasty using a digital templating system. Skeletal Radiol 2012;41:1245-9.

14. Petretta R, Strelzow J, Ohly NE, et al. Acetate templating on digital images is more accurate than computer-based templating for total hip arthroplasty. Clin Orthop Relat Res 2015;473:3752-9.

Cite this article as: Dutka J, Kiepura S, Bukowczan M. Is analog preoperative planning still applicable?-comparison of accuracy of analog and computer preoperative planning methods in total hip arthroplasty. Ann Transl Med 2021;9(9):749. doi: 10.21037/ atm-20-7489 